\title{
Determination of Permissive Cellular Lines to the Canine Herpes Virus, by Visualization of the Cytopathic Effect
}

\author{
Saldías L Navarro C* \\ Department of Animal Preventive Medicine, Chile
}

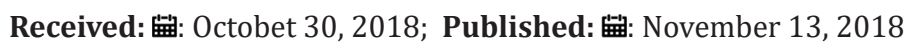

*Corresponding author: Saldías L Navarro C, Department of Animal Preventive Medicine, FAVET, University of Chile, Chile

\section{Opinion}

The viral isolation in cells in vitro is the test that presents greater sensitivity and reliability in the current virology, for this it is necessary to consider the speed of viral detection and the sensitivity of the cells to the virus. It is world-wide known that Canine herpesvirus 1 (CaHV1) despite belonging to the subfamily Alphaherpesvirinae presents a narrow range of cells in which it multiplies in the laboratory, being restricted only to cells of canine origin. The objective of this work was to look for alternative cell lines to those of canine origin whose use in the laboratory is habitual and do not present major complications in their maintenance. The research was conducted in cell lines commonly used in the Public Health Institute of Chile for the diagnosis and monitoring of diseases of impact in the country, in this case: L20b, Hep-2, MNA, $\mathrm{RD}$, Vero, MDCK. During the first stage, maintenance of the cell lines, standardization of the protocol was the critical point, since each line presents different maintenance requirements (ATCC, 2018) [1]. During the first five days of observation the MDCK and Vero lines formed the monolayer more quickly. In contrast, line L20b was the slowest and showed a tendency to discard on the fourth day, coinciding with the recommendations of the ATCC that advise to change the culture medium every two or three days. These recommendations were not followed since a common maintenance protocol was used in all the lines and this could explain the difficulty that there was for the formation and maintenance of the monolayer.

During the process of freezing / thawing the cell stock, a difficulty to recover the cells after freezing was evidenced. Initially, only fetal bovine serum was used as a cryoprotective agent, which probably did not adequately protect the cells from intracellular ice formation and osmotic changes that generated cell lysis and therefore impossible to recover them for the formation of monolayers. cell phones. When it was decided to use the protocol that included the use of DMSO as a cryoprotective agent, the recovery of cells for planting was positive and monolayer formation was achieved. Finally, the objective of this stage was fulfilled, and the cells were maintained for the next stage of viral inoculation. The viral inoculation stage was fulfilled following the protocols already described in materials and methods. From the beginning there were differences between the different lines, since training was not achieved uniform of the monolayer at $24 \mathrm{~h}$. This would probably be explained because each line had a different mitotic index, consequently they had different monolayer formation times. Also, the conditions in which the monolayer of cells is formed can vary from one cell line to another, one way to have solved this would be to plant more cells per ml. In those lines that have a slower growth or have given the ideal conditions per cell line recommended by the ATCC, however given the characteristics of the test, it was required that the conditions were uniform in all the lines, likewise cannot rule out associated variations to the manipulation given by the experience in the laboratory.

On the second day after infection with CaHV1, the first differences were observed in the culture medium and the characteristics of the monolayer, both between cell lines, and between challenge and control. The variations of tonality in the culture medium indicated that Vero and MNA presented yellow media which indicated greater acidity caused by a higher cellular metabolism. However, these differences could not be attributed to viral action because they were observed in the control and challenge bottles. The maintenance and inoculation of a greater number of bottles per cell line would have been adequate, however there were economic limitations and cellular availability that prevented maintaining a greater number of samples per cell line. On the fourth day post-infection with Canine Herpes Virus the changes were more relevant, MDCK presented differences with respect to the control, greater amount of refringent cells and spaces between the cells. The other line that presented 
differences between the challenge and control was the Vero cell line, however these differences could not be attributed with certainty to CaHV1. The lines L20b, MNA, RD presented detachment of cells in control and challenge, probably attributable to the shortage of nutrients in the environment, a situation like that which occurred in the stage of cellular maintenance prior to inoculation.

Possible explanations to the results in these lines of noncanine origin, can be related to the fact that the particle manages to penetrate the cell but does not complete the replication process and therefore does not generate an effect in the cell, that the change observed in the cell is only product of cellular aging, due to inadequate management of the necessary conditions in the maintenance of the lines or because the recommendations of the ATCC were ignored so that all the lines were under the same conditions. Finally, on the fifth day of observation the MDCK line showed a cytopathic effect attributable to CaHV1 and the Vero line also presented changes between challenge and control, but it was difficult to classify them qualitatively as attributable to the action of CaHV1 because they were not as described in the consulted literature. There is a possibility that CaHV1 does not generate

ISSN: 2574-1241

DOI: $10.26717 / B J S T R .2018 .10 .002028$

Saldías L Navarro C. Biomed J Sci \& Tech Res

This work is licensed under Creative Commons Attribution 4.0 License

Submission Link: https://biomedres.us/submit-manuscript.php the same cell lysis changes in the Vero line, since viral behavior can vary from one line to another. To verify that the changes observed in Vero are attributable to the action of CaHV1 it would have been necessary to apply virological diagnostic tests such as immunoperoxidase, enzyme immunoassay or immunofluorescence that would detect viral particles bound to the cells.

Another alternative would be to use the Polymerase chain reaction that would detect viral particles even within the cells. The results agree with the literature, which indicate that CaHV1 despite having a close relationship with other members of the Alphaherpesvirinae subfamily such as pseudorabies virus, has a very narrow range of host cells and is restricted only to cells of origin canine, whether they are primary culture or stable cell line. However, the changes that the Vero line presented during the experiment should be considered in subsequent confirmatory studies.

\section{References}

1. (2018) ATCC. American Type Culture Collection.

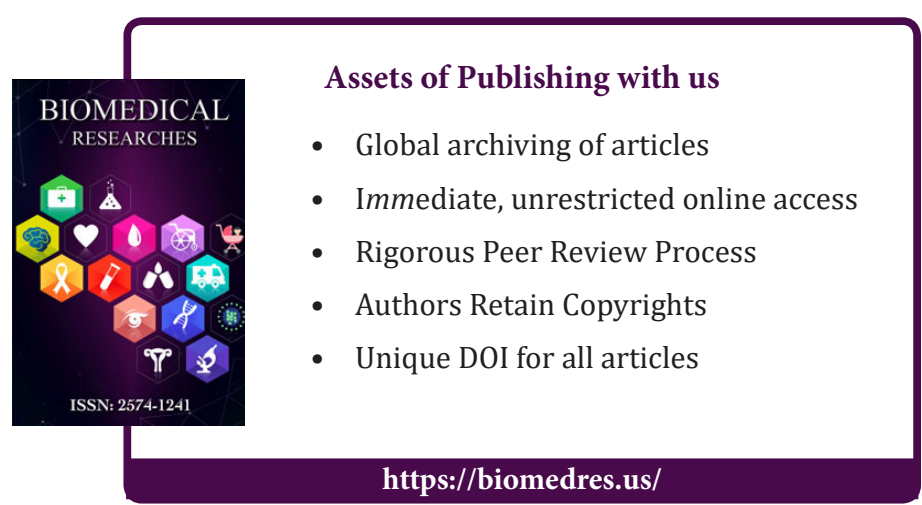

